\title{
INFLUENCIA TRANSVERSAL DE LOS COMMODITIES MINEROS EN LA ECONOMÍA PERUANA BAJO UN ENFOQUE GLOBAL
}

\author{
CROSS-CUTTING INFLUENCE OF MINING COMMODITIES ON THE PERUVIAN \\ ECONOMY UNDER A GLOBAL APPROACH
}

Nicko A. Gomero GonZales Universidad Nacional Mayor de San Marcos Lima, Perú

ORCID: https://orcid.org/0000-0002-5642-8298 Correo electrónico: ngomerog@unmsm.edu.pe

\section{RESUMEN}

Objetivo: Analizar cómo la actividad minera tiene impactos transversales en la economía, incluido el financiero. Método: Descriptivo, correlacional-causal, utilizando para ello información histórica, recopilada de fuentes oficiales, como el Banco Central de Reserva del Perú (BCRP), Comisión Chilena del Cobre (Cochilco), Bolsa de Valores de Lima (BVL). Resultados: El estudio evidencia que la minería ejerce influencia en el crecimiento económico (PBI), en la fortaleza tributaria, específicamente a través del impuesto a la renta que pagan las empresas mineras, dinamiza y profundiza el mercado de capitales a través de la negociación de acciones y las cotizaciones de estos activos financieros en el mercado bursátil, cuya volatilidad depende del comportamiento del precio de los commodities mineros. Conclusión: La minería, a pesar de los pasivos ambientales que genera, tiene efectos directos y transversales en la economía coadyuvando al proceso de crecimiento sostenido, resultado que se va a traducir en generar fortalezas en los mercados globalizados.

Palabras clave: PBI; commodities mineros; rentabilidad; tributación; acciones mineras.

\begin{abstract}
Objective: To analyze how mining activity has transversal impacts on the economy, including financial impacts. Method: Descriptive, correlational-causal, using historical information collected from official sources such as Central Reserve Bank of Peru (CRBP), Chilean Copper Commission (CHICOCO), Lima Stock Exchange (LSE). Results: The study shows that mining has an influence on economic growth (GDP), on tax strength, specifically through the income tax paid by mining companies, and that it stimulates and deepens the capital market through the trading of shares and the prices of these financial assets in the stock market, whose volatility depends on the behavior of the price of mining commodities. Conclusion: Mining, in spite of its environmental liabilities, has direct and transversal effects on the economy, contributing to the process of sustained growth. A result that will translate into the strengthening of globalized markets.
\end{abstract}

Keywords: GDP; mining commodities; profitability; taxation; mining shares.

(c) Los autores. Este artículo es publicado por la revista Quipukamayoc de la Facultad de Ciencias Contables, Universidad Nacional Mayor de San Marcos. Este es un artículo de acceso abierto, distribuido bajo los términos de la licencia Creative Commons Atribución 4.0 Internacional (CC BY 4.0) [https://creativecommons.org/licenses/ by/4.0/deed.es] que permite el uso, distribución y reproducción en cualquier medio, siempre que la obra original sea debidamente citada de su fuente original. 


\section{INTRODUCCIÓN}

La actividad minera, vía exportaciones, se ha constituido como uno de los pilares básicos que en cierta forma ha generado sostenibilidad a la economía peruana, hecho que ha quedado evidenciado con el aporte tributario, generación de divisas, fortalecimiento del mercado laboral, profundización del mercado de valores, y en términos generales en el potenciamiento del producto bruto interno (PBI). Es conveniente resaltar que, si un país robustece su sector externo, logrará resultados importantes en las cuentas macroeconómicas; para ello, como señalan Krugman y Obstfeld (2006), se necesita corregir las fallas estructurales que se pueden encontrar en el sector para lograr mejores posicionamientos en los mercados.

Las economías en vías de desarrollo están ancladas al comportamiento del escenario internacional; sin embargo, es un reto romper estos paradigmas para afianzar el crecimiento y desarrollo. Es necesario estar por encima de la teoría de las ventajas comparativas, atribuibles a Ricardo, D. (1817), para que la oscilación de los precios de los commodities no se convierta en una amenaza para la economía. En este sentido es preciso señalar a Contreras y Gutiérrez (2016), que afirman:

Los ciclos expansivos de precios de commodities son periodos determinantes para las economías de países productores de metales. En estos países, la importancia de los sectores extractivos puede medirse de diversas formas, como el peso de las divisas generadas, exportaciones o contribución directa al Producto Bruto Interno (PBI). (p.1)

Es cierto que esta actividad genera pasivos que no han sido mitigados por las empresas, pero también es cierto que los procesos de producción han mejorado y han contribuido a amortiguar los problemas ambientales. "La responsabilidad compartida obliga a construir entre todos los actores una visión de país, una visión de desarrollo y una visión de sostenibilidad" (Equipo MMSD América del Sur, 2002, p.13). Los beneficios de las buenas prácticas mineras no solo se reflejan en el escenario macroeconómico y social, sino también en las cuentas microeconómicas, por ello las empresas de este tipo están bien empoderadas en los mercados, como es el caso del bursátil, cuyas acciones marcan tendencia de los índices bursátiles. Sobre la sostenibilidad y el trabajo colaborativo de las empresas mineras, Narrea (2018) explica:

Bajo el nuevo marco internacional de las industrias extractivas, las compañías mineras son más proclives a colaborar con el gobierno, la comunidad y otras partes interesadas en desarrollar políticas que tengan como objetivo generar spillovers de conocimientos e innovaciones en la cadena de valor minera y hacia otras industrias. (p.21)

Una de las limitaciones que tiene este sector es que su actividad está supeditada al performance del mercado internacional, cuya volatilidad marca tendencia de los precios. Como bien se conoce China, especialmente por su potencial productivo, se ha constituido como el principal demandante de concentrados mineros, como es el cobre, por ello el comportamiento de su PBI o su actividad económica es gravitante para que las economías con ventajas comparativas en estos tipos de producto tengan logros importantes en su economía. Sobre la importancia de China, se señala:

La tendencia a la baja fue el resultado de una disminución de la demanda de China, debida a las tensiones comerciales entre China y los Estados Unidos, que crearon condiciones adversas para la economía y causaron una ralentización del consumo, y el aumento de las existencias en los almacenes de la Bolsa de Metales de Londres. (Naciones Unidas, 2019, p.10)

Dada las características de los mercados globalizados, una pequeña perturbación podría originar una oleada de crisis generalizada con los efectos perniciosos que este acarrea. No interesa donde y como se dé el origen de la perturbación, lo relevante es que el efecto contagio es inmediato en todas las economías; es justamente lo que viene pasando con la crisis sanitaria, que ha trastocado las estructuras económicas de los países más sólidos del mundo. En ese sentido, Delgado y Quiñón (2020) afirman que: "El Instituto de Ingenieros de Minas del Perú (llMP) estima que el PBI minero en el Perú podría descender entre 4\% y $20 \%$ en el 2020, siendo un aspecto importante el valor de las exportaciones" (p.8). Bajo este escenario de crisis, se busca conocer qué papel se le asigna al Estado para asegurar la continuidad de la actividad minera, para explicar ello se recurre a Díaz (2018), quien explica: "Existen variables que influyen en la eficiencia de la exportación de concentrados de minerales en el Perú, pero no todos pueden ser manejados por las empresas, por lo que tienen que depender del Estado" (p.8). Los países más vulnerables son los que sienten con mayor fuerza los impactos de la crisis, hecho que ha quedado evidenciado con los efectos directos y colaterales producidos por el COVID-19.

Como es claro, las cifras macroeconómicas de la economía peruana, incluidas las financieras, están influenciadas por la actividad minera; es por ello que, ante cualquier movimiento de los mercados, los impactos serán significativos y su dirección dependerá del tipo de sensibilidad que presente el precio de los commodities. Es importante que los ejecutores de las políticas económicas y 
los del nivel estratégico de las empresas sepan dar lectura al movimiento de los precios. Por el lado del gobierno, debe entenderse que los logros fiscales y la generación de divisas dependerán de la volatilidad y del grado de correlación de los precios de los minerales, y en cuanto a las empresas, se debe tener claro que la incidencia o impacto se dará por los indicadores de rentabilidad, tales como la utilidad por acción (UPA), rendimiento del capital (ROE), rentabilidad sobre los activos (ROA), ingresos, y/o utilidad neta. Parafraseado a Pacheco (2018) se puede afirmar que, a través del comportamiento del ROE de una empresa minera para un periodo determinado, se hacen implícitos los comportamientos cíclicos de los precios de los minerales, cuyo impacto además afecta a los flujos financieros.

En función a lo señalado, la problemática del estudio se centra en la elevada importancia que tiene la actividad minera en el comportamiento de la economía peruana, tanto macro y microeconómicamente. Esto incluye también a la posición financiera de las empresas que operan en este sector y la inestabilidad de los mercados internacionales, que al mismo tiempo generan volatilidades en los precios de los commodities y afectan la sostenibilidad de la economía. Por ello, el objetivo del estudio se centra en explicar la manera en la cual la actividad minera genera impactos transversales en la economía, y la relación que se aprecia entre los precios de los commodities con el performance de la economía de China y de EE. UU, las cuales ejercen alta influencia en estos mercados. Además, se plantea como hipótesis que la cotización de los precios de los commodities mineros ejercen influencia en el precio de la acción de la empresa minera peruana Buenaventura, cuya fortaleza se centra en la exportación de metales preciosos al mercado internacional.

\section{MATERIALES Y MÉTODOS}

La investigación fue cuantitativa, descriptiva, causal y no experimental. Se utilizó información secundaria de fuentes oficiales, como el Banco Central de Reserva del Perú (BCRP), Comisión Chilena del Cobre (Cochilco), Bolsa de Valores de Lima (BVL), lo que da mayor fiabilidad a los resultados del estudio. Se estructuró la matriz de correlación con el coeficiente de Pearson del portafolio de productos mineros; asimismo, se contrastó el precio de los commodities mineros con el PBI de China y EE. UU. Por último, se aplicó el modelo logarítmico $(\log =\log )$ para determinar la incidencia de los precios de los metales preciosos en el valor de la acción de la empresa minera peruana Buenaventura, que exporta oro y plata y a la vez cotiza en la Bolsa de New York y en la Bolsa de Valores de Lima. El modelo $\log =$ Log es el siguiente: $\operatorname{LnYi}=B o+B_{1} \operatorname{LnX} 1 \mathrm{i}+$ $B_{2} \operatorname{LnX} 2 \mathrm{i}+\mathrm{Ui}$, con un $95 \%$ de confiabilidad, donde $B_{o}, B_{1}$ $y B_{2}$ son los betas de la ecuación que miden la sensibilidad de la acción de BNV ante un cambio en el precio de los minerales en el mercado internacional.

\section{RESULTADOS}

La globalización de los mercados ha profundizado aún más la sensibilización de los minerales, cuya volatilidad, en la mayoría de los casos, presenta el mismo recorrido y puede ser corroborado con el coeficiente de correlación (r), cuyo resultado da una clara señal de las asimetrías del portafolio de minerales más demandados en el mercado internacional. Como se conoce, China, por su hegemonía económica, es la economía que mayor dinamización le da a este mercado. Por ejemplo, en el caso del cobre, este país muestra su liderazgo consumiendo el 50\% de este producto que se negocia en el mercado mundial, siendo uno de sus proveedores el Perú, abasteciéndole el $27 \%$ de este concentrado. China, por su dinámica productiva, no solo posee una alta participación en la importación de cobre, sino en casi todo el portafolio de minerales que exporta la economía peruana; como es el zinc, molibdeno y hierro, que, según el Ministerio de Energía y Minas (MINEM, 2018), aportan en promedio el $70 \%$ del valor de las exportaciones totales. El MINEM (2018) señala lo siguiente: "En el ranking mundial, Perú se mantuvo como el segundo mayor productor de cobre, plata y zinc; asimismo, se posicionó en el tercer lugar como productor de estaño, cuarto lugar como productor de molibdeno y sexto como productor de oro" (p. 26). El empoderamiento de la economía peruana con la exportación de minerales es una clara señal de la forma en la que se ha desarrollado esta industria en el Perú, respaldado por los flujos de inversiones mayormente de procedencia extranjera. En cuanto a este punto en la Memoria del MINEM (2018) se señala lo siguiente:

Así, desde que se tiene información registrada en la base de datos del Ministerio de Energía y Minas (1996) hasta el 2018, la inversión reportada por los titulares de la actividad minera asciende a US\$ 65,273 millones y solamente durante la última década se ejecutó el $85.1 \%$ debido al desarrollo de proyectos mineros de gran magnitud. (p.68)

El flujo de inversiones potencializa todo el ciclo productivo de la minería, que va desde la exploración hasta la comercialización, el cual, vía efectos multiplicadores, generará impactos positivos en los demás mercados, como el laboral. Sobre este punto cabe mencionar un informe de empleo minero presentado por el MINEM (2019) en donde, haciendo referencia al Instituto Peruano de Economía (IPE), llega a precisar que por cada empleo generado de 
forma directa en la actividad minera, se generan adicionalmente 6.25 empleos en el resto de la economía.

Todas estas características se ven complementadas con el aporte de esta actividad en la formación del PBI que, según las cifras de Instituto Peruano de Economía (IPE, 2018), es del $10 \%$. Tal como se aprecia en la figura 1, la correlación entre estas dos variables es directa $\mathrm{r}=$ $11.92 \%$, lo cual corrobora que las exportaciones inciden directamente en la formación del PBI. Los impactos macroeconómicos; además, pasan por el aporte de la actividad minera en la recaudación tributaria, que dicho sea de paso coadyuvan a robustecer la presión tributaria. Al contrastar el aporte tributario de las empresas mineras con el total de impuestos que se recaudan por tercera categoría, se aprecia una importante participación con un pico del $15 \%$ para el 2018, tal como se aprecia en la tabla 1. Esta contribución tributaria depende de las oscilaciones de los precios de los commodities mineros en el mercado internacional.
Los impactos no solo están en el sector real de la economía, sino también en el financiero; por ello, las acciones mineras marcan tendencias de los índices bursátiles y profundizan el mercado de valores, mercado cuyo monto negociado en el 2020, según un reporte de la Bolsa de Valores de Lima (2020), llegó a los 577502 soles, cifra que representa el $5 \%$ de lo negociado en acciones en el referido año que llegó a los 12222156 miles de soles. El grado de liquidez es otra de las características de estos activos financieros; para evidenciar lo afirmado se cita un reporte de la BVL sobre este indicador, donde se puede apreciar que Volcán y Buenaventura, por ejemplo, son empresas mineras que están en los primeros puestos del ranking de liquidez, con 14,81 y 12,18 respectivamente. En la tabla 2 se aprecia la participación de las acciones mineras en el total de acciones negociadas en la Bolsa de Valores de Lima.

A continuación, se hará un análisis de un portafolio de productos mineros, en el cual el Perú posee fortalezas

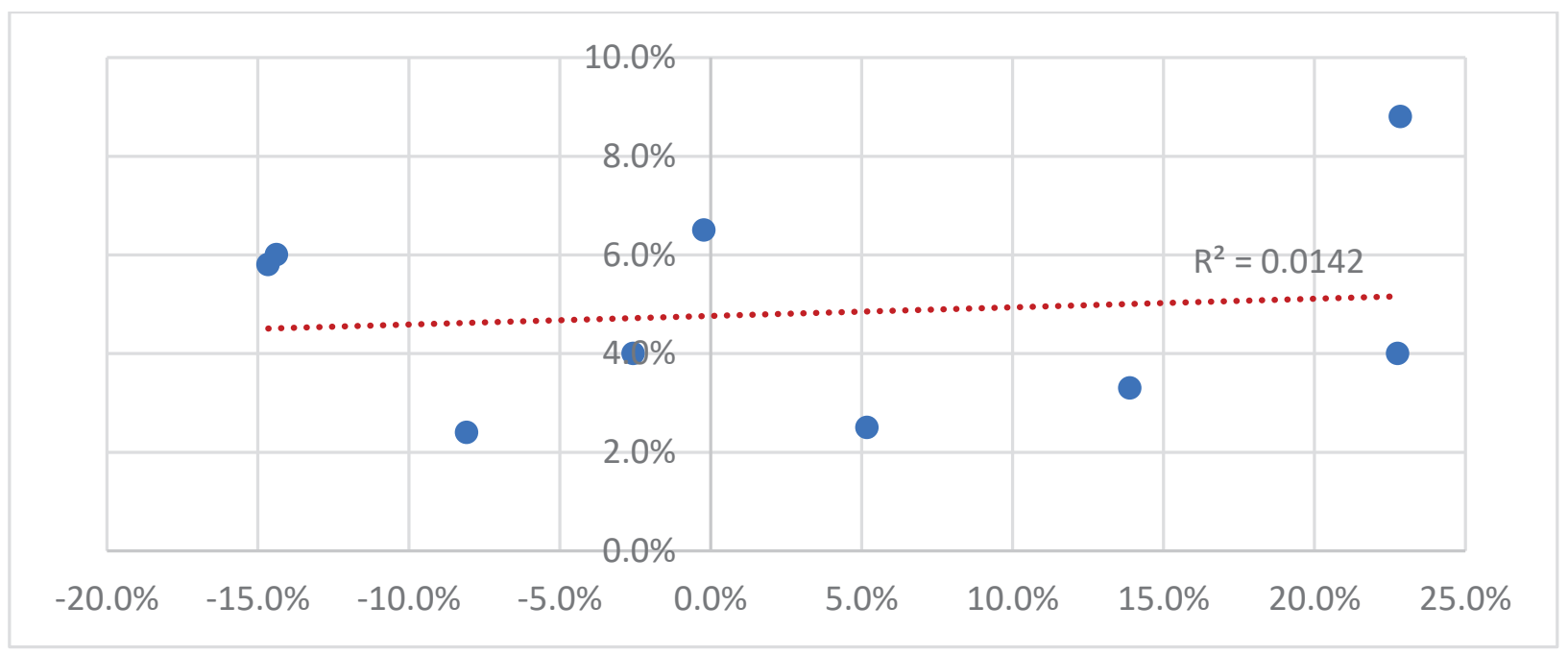

Figura 1. Exportaciones mineras y PBI. (2011-2019) variación porcentual

Fuente: Elaboración propia con información de OSINERGMING y BCRP

Tabla 1

Impuestos a la renta de tercera categoría: Minería 2016-2019 (millones de soles)

\begin{tabular}{cccc}
\hline Años & Minería & Total, tercera Categoría & Imp. Minería/total impuesto renta tercera categoría \\
\hline 2016 & 770 & 16496 & $4.7 \%$ \\
2017 & 1727 & 15499 & $11.1 \%$ \\
2018 & 2585 & 17268 & $15.0 \%$ \\
2019 & 2036 & 17679 & $11.5 \%$ \\
\hline
\end{tabular}

Fuente: Elaboración propia con información de Memoria 2019, BCRP, 2019. 
productivas y exportadoras, como es el cobre, y se determinará el grado de correlación de los precios de los productos, tal como se aprecia en la tabla 3, para los años analizados que va de 1999 al 2020. Todos los precios van en la misma dirección, lo cual es corroborado con el coeficiente de correlación de Pearson, destacando el resultado para los metales preciosos debido a que este coeficiente llega al 89,9\%, presentándose además una fuerte correlación entre el cobre, el oro y la plata, coeficiente que llega al $81,7 \%$ y $87,5 \%$, respectivamente. Tal como se evidencia en la tabla 3 , el coeficiente de Pearson en el contraste de precios en su mayoría supera al 70\%, con excepción del zinc y la plata que alcanza el 50,2\%. Estas cifras son importantes para la economía peruana, ya que es mayor el dinamismo del mercado de commodities y con ello el repunte de los precios incidirá favorablemente para robustecer el proceso de crecimiento económico, pasando a impulsar la Bolsa de Valores.

No se puede dejar de lado que, ante un retroceso de la economía global y con ello el declive económico de las principales economías del mundo, dado el grado y tipo de correlación, los impactos negativos de tipo económico y financiero serán significativos, hecho que se evidencia cuando los Índices Bursátiles retroceden ante caídas del precio de los minerales.

Se ha comentado que la economía China, por su fortaleza económica a nivel global, ejerce presión a los precios de los commodities, cuyas oscilaciones o comportamientos cíclicos guardan correlación con el PBI de este país y en segundo orden de la economía norteamericana. Hay que tener presente que la suma del valor de la producción de

Tabla 2

Monto negociado de las acciones mineras en la BVL 2016-2020 (miles de nuevos soles)

\begin{tabular}{lccccc}
\hline & $\mathbf{2 0 1 6}$ & $\mathbf{2 0 1 7}$ & $\mathbf{2 0 1 8}$ & $\mathbf{2 0 1 9}$ & $\mathbf{2 0 2 0}$ \\
\hline Total acciones & 8615421 & 20073499 & 10739661 & 11688799 & 12222156 \\
Acciones de capital mineras & 1429799 & 3935948 & 812762 & 414244 & 415646 \\
Acciones de inversión mineras & 148261 & 270349 & 118588 & 178532 & 161856 \\
Total acciones Mineras & 1578060 & 4206297 & 931350 & 592776 & 577502 \\
Acciones mineras/total acciones & $18.3 \%$ & $21.0 \%$ & $8.7 \%$ & $5.1 \%$ & $4.7 \%$ \\
\hline
\end{tabular}

Fuente: Elaboración propia con información de Informe Mensual, Bolsa de Valores de Lima, 2020.

Tabla 3

Matriz de correlación de precios de productos mineros: 1999-2020 (US\$)

\begin{tabular}{|c|c|c|c|c|c|}
\hline & & Cobre & oro & plata & zinc \\
\hline \multirow[t]{3}{*}{ Cobre } & Correlación de Pearson & 1 &, $817^{\star *}$ & $875^{\star *}$ &, $784^{* *}$ \\
\hline & Sig. (bilateral) & &, 000 &, 000 & ,000 \\
\hline & $\mathrm{N}$ & 22 & 22 & 22 & 22 \\
\hline \multirow[t]{3}{*}{ Oro } & Correlación de Pearson &, $817^{* \star}$ & 1 &, $899 * *$ &, $569^{* *}$ \\
\hline & Sig. (bilateral) &, 000 & &, 000 & ,006 \\
\hline & $\mathrm{N}$ & 22 & 22 & 22 & 22 \\
\hline \multirow[t]{3}{*}{ Plata } & Correlación de Pearson &, $875^{\star \star}$ &, $899^{* *}$ & 1 &, $502^{*}$ \\
\hline & Sig. (bilateral) &, 000 &, 000 & &, 017 \\
\hline & $\mathrm{N}$ & 22 & 22 & 22 & 22 \\
\hline \multirow[t]{3}{*}{ Zinc } & Correlación de Pearson &, $784^{* *}$ &, $569^{\star *}$ &, $502^{*}$ & 1 \\
\hline & Sig. (bilateral) & ,000 & ,006 & ,017 & \\
\hline & $\mathrm{N}$ & 22 & 22 & 22 & 22 \\
\hline
\end{tabular}

**. La correlación es significativa en el nivel 0,01 (2 colas).

*. La correlación es significativa en el nivel 0,05 (2 colas).

Fuente: Elaboración propia con información de Base de datos de minerales, Comisión Chilena del Cobre (Cochilco), 2020. 
ambos países aporta el 45,2\% de la producción global, esta cifra sustenta la elevada influencia de estas economías en los diferentes mercados y distintos productos a nivel global.

Los coeficientes de correlación que se presentan en la tabla 4 dan una lectura de la influencia de las principales economías del mundo, especialmente de la China en el precio de los metales y también la forma como la economía peruana está anclada a estos componentes exógenos. Al contrastar el PBI de China con el precio del cobre y del oro, arroja una correlación del 52.4\% y del $50.9 \%$ respectivamente, cifras que dan señales del grado y del tipo de influencia de esta economía en el precio de estos dos commodities mineros en el mercado internacional; por ello, es importante para la economía peruana que el PBI chino experimente altas tasas de crecimiento, situación que se vería reflejada en los indicadores macroeconómicos y en una mayor fortaleza financiera de las empresas mineras. El hecho de que la correlación entre el precio del cobre y del oro con el PBI de Perú llega al $32,6 \%$ y $11,4 \%$ respectivamente, le da consistencia a la afirmación sobre el efecto positivo que podría ejercer el precio de estos minerales en este indicador macroeconómico, el cual es el punto de partida para la mejora de otros indicadores de crecimiento económico. Para la economía peruana no solo es importante el crecimiento del precio de los Commodities mineros, sino también es de relevancia que las dos economías más importantes del mundo, dado los resultados de correlación, como es el caso con el PBI de China el cual llega al 59.7\% y para EE. UU. del $63.15 \%$, presenten cifras sostenidas de crecimiento económico. Las cifras presentadas en la tabla 4 dan señales claras de la relación directa que existe entre los precios de los minerales, como es el cobre y el oro, con el performance de las economías más importantes del mundo, y que además van a ejercer dinamismos a la economía peruana.

Un hecho de relevancia es el comportamiento del PBI de China y EE. UU. Esta primera economía ha tenido un crecimiento significativo, ubicándose como la segunda economía más grande del mundo; sin embargo, la economía norteamericana sigue siendo la más fuerte del globo, tal como lo señala JP Morgan, quien considera que el aporte de la economía norteamericana al PBI global es del 27,3\% y el del país asiático es del 17,9\% (Nieves, 2020).

Como se conoce, la crisis sanitaria, aparte de generar problemas severos en los indicadores de salud, ha trastocado los cimientos de la economía global, vulnerando

Tabla 4

Matriz de correlación de precios de productos mineros, PBI de China, EE. UU y Perú 2000 -2020 (variación \%)

\begin{tabular}{|c|c|c|c|c|c|c|}
\hline & & Pcobre & Poro & Pchina & Peeuu & Pperú \\
\hline \multirow[t]{3}{*}{ Pcobre } & Correlación de Pearson & 1 & $494^{*}$ &, $524^{*}$ & $444^{*}$ & ,326 \\
\hline & Sig. (bilateral) & & ,023 & 015 &, 044 & , 150 \\
\hline & $\mathrm{N}$ & 21 & 21 & 21 & 21 & 21 \\
\hline \multirow[t]{3}{*}{ Poro } & Correlación de Pearson &, $494^{*}$ & 1 &, $509^{*}$ &,- 273 & ,114 \\
\hline & Sig. (bilateral) & ,023 & & ,018 & ,231 & ,624 \\
\hline & $\mathrm{N}$ & 21 & 21 & 21 & 21 & 21 \\
\hline \multirow[t]{3}{*}{ Pchina } & Correlación de Pearson &, $524^{*}$ &, $509^{*}$ & 1 & ,212 &, $597^{* *}$ \\
\hline & Sig. (bilateral) & ,015 &, 018 & & ,357 &, 004 \\
\hline & $\mathrm{N}$ & 21 & 21 & 21 & 21 & 21 \\
\hline \multirow[t]{3}{*}{ Peeuu } & Correlación de Pearson & $444^{*}$ &,- 273 & ,212 & 1 &, $631^{* *}$ \\
\hline & Sig. (bilateral) &, 044 &, 231 &, 357 & &, 002 \\
\hline & $\mathrm{N}$ & 21 & 21 & 21 & 21 & 21 \\
\hline \multirow[t]{3}{*}{ Pperu } & Correlación de Pearson & ,326 & ,114 &, $597^{\star \star}$ &, $631^{* *}$ & 1 \\
\hline & Sig. (bilateral) & , 150 & ,624 &, 004 & ,002 & \\
\hline & $\mathrm{N}$ & 21 & 21 & 21 & 21 & 21 \\
\hline
\end{tabular}

*. La correlación es significativa en el nivel 0,05 (2 colas).

**. La correlación es significativa en el nivel 0,01 (2 colas).

Fuente: Elaboración propia con información de Base de datos de minerales, Comisión Chilena del Cobre, 2020, y Perspectivas de la Economía Mundial, Fondo Monetario Internacional, 2020. 
el proceso de crecimiento económico, especialmente en las economías más débiles, por ello el quiebre de los diferentes mercados. Para dar una lectura a la gravedad de la crisis, es necesario analizar la información proporcionada por el Fondo Monetario Internacional (FMI, 2020) en donde se observa la caída significativa de los PBI de las economías avanzadas y de los países emergentes, excepto el de China, cuyo crecimiento económico estimado llegaría al 1,7\%. Sin duda los efectos perniciosos de la crisis son severos, bastó un año para trastocar los cimientos de la economía global, volatilizándose con ello el mercado de commodities. La correlación de precios mensuales que se observa en la tabla 5 , que incluye el periodo de la pandemia, a diferencia de las cifras anuales, proyectan un mayor grado de relación y de tipo directa. La correlación ( $\mathrm{r}$ ) entre los metales preciosos llega al 82,4\%, siendo más significativa para el cobre y el estaño, la cual llega al 98,8\%; además, se aprecia el cobre y el zinc, la cual llega al 93,5\%. En época de pandemia, los precios de los commodities se acomodaron con mayor fuerza y tuvieron el mismo comportamiento, señalando estos resultados para el portafolio de productos mineros, y la oscilación de sus precios fue mucho más estrecha que en los periodos anuales, generando por ello mayores incidencias negativas en los indicadores macro y microeconómicos, hecho que en parte sustenta la fuerte caída del PBI de la economía nacional que según el Instituto Nacional de
Estadística e Informática (2020) al tercer trimestre del 2020 con relación al 2019, llegaba a una caída acumulada del $-14,5 \%$, con una contracción de las exportaciones del $-23,7 \%$.

También es importante evaluar la manera cómo la volatilidad del precio de los minerales ha incidido en el precio de las acciones. Se conoce que el mercado de capitales en parte está influenciado por el precio de los minerales, especialmente en aquellos países cuyo mercado bursátil experimenta una alta dependencia a estos tipos de productos. Para este propósito se ha contrastado el precio de las acciones de Buenaventura que cotiza en la Bolsa de New York y el precio del oro y la plata, cuyos productos poseen alta ponderación en su portafolio de exportaciones. En función de los datos contrastados, en el periodo de enero 2018 a diciembre 2020, el coeficiente de correlación múltiple es del 59,4\% y al estructurar la ecuación logarítmica se aprecia que el precio de las acciones reaccionaron, incluido el periodo de crisis sanitaria, en forma contraria al comportamiento del precio del oro, hecho que se sustenta por el $B_{1}:-1,37$; es decir, ante un cambio en un dólar en el precio del oro, las acciones cambiaron en forma contraria en 1,37 dólares, caso contrario sucedió con la plata, que por cada dólar que cambiaba el precio de las acciones de la empresa se sensibilizaba en 0,647 centavos, siendo el cambio en la misma dirección. Por ello se observa que, las

Tabla 5

Matriz de correlación de precios de los minerales en época de crisis sanitaria: diciembre 2019 -octubre 2020

\begin{tabular}{|c|c|c|c|c|c|c|}
\hline & & cobre & oro & plata & zinc & estaño \\
\hline \multirow[t]{3}{*}{ cobre } & Correlación de Pearson & 1 & 570 & $881^{* *}$ & $935^{* *}$ & ,988** \\
\hline & Sig. (bilateral) & &, 067 &, 000 &, 000 &, 000 \\
\hline & $\mathrm{N}$ & 11 & 11 & 11 & 11 & 11 \\
\hline \multirow[t]{3}{*}{ oro } & Correlación de Pearson &, 570 & 1 & $824^{* *}$ & ,406 &, 504 \\
\hline & Sig. (bilateral) & ,067 & &, 002 & ,215 & ,114 \\
\hline & $\mathrm{N}$ & 11 & 11 & 11 & 11 & 11 \\
\hline \multirow[t]{3}{*}{ plata } & Correlación de Pearson &, $881^{* *}$ & ,824* & 1 & $828^{* *}$ &, $834^{* *}$ \\
\hline & Sig. (bilateral) &, 000 &, 002 & &, 002 & 001 \\
\hline & $\mathrm{N}$ & 11 & 11 & 11 & 11 & 11 \\
\hline \multirow[t]{3}{*}{ zinc } & Correlación de Pearson &, $935^{* *}$ &, 406 & $828^{* *}$ & 1 &, $918^{* *}$ \\
\hline & Sig. (bilateral) &, 000 & ,215 &, 002 & & ,000 \\
\hline & $\mathrm{N}$ & 11 & 11 & 11 & 11 & 11 \\
\hline \multirow[t]{3}{*}{ estaño } & Correlación de Pearson &, $988^{* *}$ &, 504 &, $834^{* *}$ & $918^{* *}$ & 1 \\
\hline & Sig. (bilateral) &, 000 & ,114 & ,001 &, 000 & \\
\hline & $\mathrm{N}$ & 11 & 11 & 11 & 11 & 11 \\
\hline
\end{tabular}

Nota: La correlación es significativa en el nivel 0,01 (2 colas).

Fuente: Elaboración propia con información de Base de datos de minerales, Comisión Chilena del Cobre, 2020. 
acciones de Buenaventura mostraron mayor sensibilidad a los cambios de los precios del oro en el mercado de commodities (Ver Tablas 6-8).

Cabe precisar que el precio del oro y de la plata, que actúan como variables dependientes por el resultado del Pvalor que es del $0.000 \%$ menor al 5\%, resultado para el modelo general, corrobora la hipótesis que indica que el comportamiento del precio de las acciones de Buenaventura en el periodo de estudio es explicada o influenciada por el precio de los commodities, que van a incidir en el valor de la empresa y generar oportunidades de rentabilidad a los que estructuran portafolios financieros con estos tipos de activos.

Asimismo, la hipótesis por variable queda validada debido a que el Pvalor está por debajo del 5\%, señalando que el precio de ambos minerales explica el precio de las acciones de la empresa minera.
Como se aprecia, la actividad minera genera efectos transversales: las cuentas fiscales, el PBI, el mercado de divisas, el tipo de cambio, el mercado capital, y el valor de las empresas son influenciados por el precio de los commodities mineros y por el flujo de exportaciones de estos productos, que como se evidenció presentan volatilidades por el comportamiento del mercado global.

\section{DISCUSIÓN}

Es conocido que el comportamiento de las principales economías del mundo marca tendencia, especialmente el de China y EE.U.U., cuya volatilidad se ve reflejada en los distintos mercados. Es por ello que existe gran relevancia en que estos dos países mantengan sus economías estables y en crecimiento. Es importante mencionar que estas dos economías, debido a la crisis sanitaria, también experimentaron contracciones importantes comprometiendo el

Tabla 6

Resumen del modelo

Resumen del modelo

\begin{tabular}{ccccc}
\hline Modelo & $\mathbf{R}$ & $\mathbf{R}$ cuadrado & R cuadrado ajustado & Error estándar de la estimación \\
\hline 1 &, $628^{\mathrm{a}}$ &, 395 &, 358 &, 17714 \\
\hline
\end{tabular}

Nota: Predictores: (Constante), LPplata, LPoro

Fuente: Elaboración propia

Tabla 7

Análisis de varianza

\begin{tabular}{|c|c|c|c|c|c|c|}
\hline \multicolumn{7}{|c|}{ ANOVA $^{a}$} \\
\hline & Modelo & Suma de cuadrados & gl & Media cuadrática & $\mathbf{F}$ & Sig. \\
\hline \multirow{3}{*}{1} & Regresión &, 675 & 2 & ,337 & 10,755 &, $000^{\mathrm{b}}$ \\
\hline & Residuo & 1,036 & 33 & ,031 & & \\
\hline & Total & 1,711 & 35 & & & \\
\hline
\end{tabular}

Nota: Variable dependiente: LPaccionBv. Predictores: (Constante), LPplata, LPoro

Fuente: Elaboración propia

Tabla 8

Modelo de regresión lineal múltiple: Modelo $L O G=L O G$

\begin{tabular}{|c|c|c|c|c|c|c|}
\hline \multicolumn{7}{|c|}{ Coeficientes $^{a}$} \\
\hline \multirow{2}{*}{\multicolumn{2}{|c|}{ Modelo }} & \multicolumn{2}{|c|}{ Coeficientes no estandarizados } & \multirow{2}{*}{$\begin{array}{c}\text { Coeficientes estandarizados } \\
\text { Beta }\end{array}$} & \multirow{2}{*}{$\mathbf{t}$} & \multirow{2}{*}{ Sig. } \\
\hline & & B & Error estándar & & & \\
\hline \multirow{3}{*}{1} & (Constante) & 10,766 & 1,769 & & 6,088 & ,000 \\
\hline & LPoro & $-1,373$ & ,302 &,- 874 & $-4,545$ &, 000 \\
\hline & LPplata & ,637 & ,247 & ,496 & 2,579 & ,015 \\
\hline
\end{tabular}

Nota: Variable dependiente: LPaccionBv

Fuente: Elaboración propia 
precio de los commodities mineros, ya que, como se sabe, los países que poseen ventajas absolutas en estas actividades son afectados por las volatilidades de los minerales, impacto que llega a las cuentas macro- y microeconómicas y que contrasta con lo señalado por Contreras y Gutiérrez (2016) y el IPE (2018). Los resultados de la correlación evidencian lo señalado, especialmente con el del país asiático que presenta mayor grado de relación con el precio de los minerales que oscilan de acuerdo con las coyunturas del mercado. Los resultados de la correlación y de los aportes de ambas economías a la economía global, sustentan la importancia del buen performance económico de estas economías para reestablecer las demás economías del mundo, especialmente las subdesarrolladas.

La estabilidad fiscal, la robustez de los fondos del tesoro público y la sostenibilidad del crecimiento económico, están influenciadas por la actividad minera o los ciclos de los precios y también por los indicadores financieros de las empresas mineras, lo cual contrasta con Pacheco (2018), quedando evidenciado con los resultados macroeconómicos de la economía peruana.

Por otro lado, el mercado de capitales también está supeditado a lo que suceda en el mercado de commodities, por ello la volatilidad de los precios de los minerales se va a ver reflejada en el cambio de las cotizaciones de las acciones. El impacto es diverso, unas acciones son más o menos sensibles a estos cambios, tal como sucede con Buenaventura, cuyas acciones se cotizan en la Bolsa de New York, sin perder de vista claro está, la importante participación de las acciones mineras en las negociaciones bursátiles en la BVL.

Como se puede apreciar, el impacto en la volatilidad de los precios de los minerales, el cual es influenciado por factores del mercado global, es diverso y transversal, y su incidencia puede afectar hasta los programas sociales, que como se conoce son financiados con impuestos que pagan las empresas mineras, cuya cobertura es la población más vulnerable del país.

\section{REFERENCIAS}

Banco Central de Reserva del Perú. (2019). Memoria 2019 [versión PDF]. Recuperado de: https://www.bcrp.gob. pe/publicaciones/memoria-anual/memoria-2019. html

Bolsa de Valores de Lima. (2020). Informe Mensual [versión PDF]. Recuperado de: https://finance.yahoo.com/ quote/BVN? $\mathrm{p}=\mathrm{BVN}$ \&.tsrc $=$ fin - srch

Comisión Chilena del Cobre. (2020). Base de datos de minerales. Recuperado de: https://www.cochilco.cl/Paginas/Estadisticas/Bases\%20de\%20Datos/Bases-de-Datos.aspx
Contreras, A. y Gutiérrez B. (2016). Efectos de ciclos de precios de metales: estimación de un modelo de equilibrio general con time to build para la economía peruana, 2001-2015 (Trabajo de investigación presentado para optar el grado académico de magíster en Economía). Recuperado del repositorio institucional de la Universidad del Pacífico https://repositorio.up.edu.pe/handle/11354/1210

Delgado, G. y Quiñón, A. (mayo de 2020). COVID-19 y Minería: a sesenta días de la declaratoria de estado de emergencia. Nota informativa. Recuperado de: http:// sisisemail.up.edu.pe/sisisemail/_data/2020/11950/ CEMS_Nota-informativaCV-19-001-2020.pdf

Díaz, I. (2018). Mejora de la eficiencia de exportación de concentrados de mineral en el Perú (Trabajo de fin de curso). Recuperado del repositorio institucional de la Universidad de Lima https://repositorio.ulima.edu. pe/bitstream/handle/20.500.12724/6338/Diaz_mejora_eficiencia_exportaci\%c3\%b3n_concentrados_mineral.pdf? sequence $=1$ \&isAllowed $=y$

Equipo MMSD América del Sur. (2002). Minería, minerales y desarrollo sustentable en América del Sur [versión PDF]. Recuperado de: http://www2.congreso.gob. pe/sicr/cendocbib/con4_uibd.nsf/7832DF547B40C2FF05257EF2006E308A/\$FILE/Miner\%C3\%ADa_ Minerales_y_Desarrollo_Sustentable.pdf

Fondo Monetario Internacional. (11 de octubre de 2020). Perspectivas de la Economía Mundial, octubre de 2020. Recuperado de: https://www.imf.org/es/Publications/WEO/Issues/2020/09/30/world-economic-outlook-october- 2020

Instituto de Estadística e Informática. (2020). Informe técnico. PBI. Recuperado de https://www.inei.gob.pe/ biblioteca-virtual/boletines/pbi-trimestral/1/

Instituto Peruano de Economía. (19 de abril de 2018). Aporte de la Minería al PBI. Recuperado de: https:// www.ipe.org.pe/portal/aporte-de-la-mineria-al-pbi/

Krugman, P. y Obstfeld, M. (2006). Economía Internacional. Teoría y política (7a ed.). Madrid: Pearson Educación.

Ministerio de Energía y Minas. (2018). Anuario Minero 2018. Lima: Autor.

Ministerio de Energía y Minas. (2019). Anuario Minero 2019. Lima: Autor.

Naciones Unidas. (22 de enero de 2020). Evolución reciente, retos y oportunidades en los mercados de productos básicos. Conferencia de las Naciones Unidas sobre Comercio y Desarrollo, Ginebra. Recuperado de: ht- 
tps://unctad.org/system/files/official-document/cimem2d50_es.pdf

Narrea, O. (2016). AGENDA 2030: La minería como motor de desarrollo económico para el cumplimiento de los Objetivos de Desarrollo Sostenible 8, 9, 12 y 17. Recuperado del sitio de Internet del Consorcio de Investigación Económica y social https://www.cies.org.pe/es/ investigaciones/agenda-2030-la-mineria-como-motor-de-desarrollo-economico-para-el

Nieves, V. (17 de octubre de 2020). China supera a la Eurozona como segunda economía mundial y empieza a acercarse a EE. UU. El economista.es. Recuperado de: https://www.eleconomista.es/economia/ noticias/10825322/10/20/China-supera-a-la-Eurozona-como-segunda-economia-mundial-y-empieza-a-acercarse-a-EEUU.html
Osinergmin (2020). Observatorio Energético Minero. Recuperado de: https://observatorio.osinergmin.gob.pe/ exportaciones-mineras-tradicionales

Pacheco, R. (2018). ROE y estructura financiera de las Empresas mineras periodo 2004-2013. Pensamiento Crítico, 23(2), 273 - 290.

Ricardo, D. (1817). Principios de la Economía Política y Tributación. Inglaterra: Editorial John Murray. 